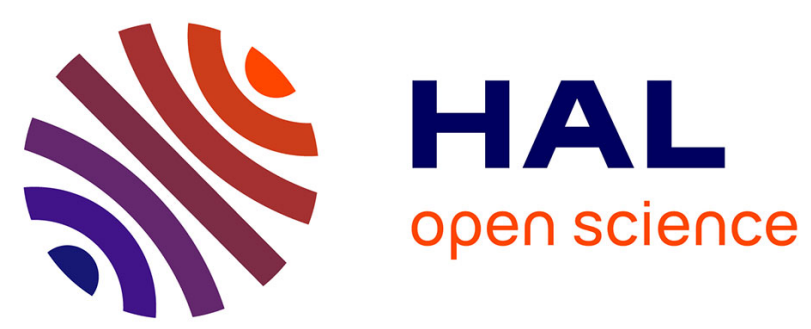

\title{
La barbaque du bareback
}

\author{
Eric Toubiana
}

\section{To cite this version:}

Eric Toubiana. La barbaque du bareback: D'une posture inconfortable. Cliniques méditerranéennes, 2010, L'imposture dans le siècle, 81, pp.141-148. 10.3917/cm.081.0141 . hal-01519434

\section{HAL Id: hal-01519434 https://hal.science/hal-01519434}

Submitted on 8 Jun 2017

HAL is a multi-disciplinary open access archive for the deposit and dissemination of scientific research documents, whether they are published or not. The documents may come from teaching and research institutions in France or abroad, or from public or private research centers.
L'archive ouverte pluridisciplinaire HAL, est destinée au dépôt et à la diffusion de documents scientifiques de niveau recherche, publiés ou non, émanant des établissements d'enseignement et de recherche français ou étrangers, des laboratoires publics ou privés. 


\section{Éric Toubiana}

\section{La barbaque du bareback D'une posture inconfortable}

Les deux termes qui nous servent aujourd'hui d'entrée en matière pour tenter de définir les avatars de notre clinique sont : masochismes et addictions, et les deux sont servis au pluriel. Deux autres mots vont les accompagner tout au long de ce propos : la barbaque et le bareback. Signifier d'emblée cette utilisation des pluriels est le fruit d'une attention toute particulière à éviter de poser le « masochisme » comme une structure à part entière mais à supporter l'hypothèse que le masochisme n'est qu'un artefact de la pulsion, une instance présente en chacun d'entre nous mais dont la configuration varie au gré des structures qu'il investit.

La chose va nous apparaître plus simple lorsqu'il s'agit d'une véritable perversion masochiste. Là, le but avoué de la pulsion est déclaré en tant que tel : se donner du plaisir en se faisant souffrir, en se faisant mal. Se faire mal n'est pas égal à se faire du mal. Se faire mal est égal à se donner du plaisir ou à trouver un objet susceptible de le faire. Là, ce sont aux relations de domination et de souffrances décrites par Sacher Masoch dans La Vénus à la fourrure que nous nous référons ${ }^{1}$. Ce type de perversion dont Sacher-Masoch est devenu le modèle trouve sa place à côté $\mathrm{d}^{\prime}$ autres perversions cataloguées dans le livre de Kraft Ebing : Psychopathia sexualis. Que ceux qui manquent d'imagination se réfèrent à cet ouvrage et leurs curiosités seront ainsi comblées. Il faut dire que nous voyons peu les types de pathologies recensés par Kraft Ebing puisque soit ces sujets s'accommodent bien de leurs fantaisies sexuelles, soit $c^{\prime}$ est devant les magistrats qu'ils auront à rendre compte de leurs « créativités sexuelles ${ }^{2}$ ».

Éric Toubiana, psychanalyste, maître de conférences, université de Paris 7 ; 87 quai de la Gare F-75013 Paris.

1. R. Sacher-Masoch, La Vénus à la fourrure, Paris, Les Mille et une nuits, 1976.

2. Krafft-Ebing (1886), Psychopathia sexualis, Paris, Payot, 1960. 
Mais Sacher Masoch a écrit un autre ouvrage qui nous éclaire bien plus sur le masochisme : Le legs de Caïn ${ }^{3}$. Caïn premier meurtrier et fratricide va être maudit par Dieu et devra, avec sa tribu, errer éternellement dans la souffrance. L'immanence de la douleur, de la souffrance châtie celui qui a osé donner la mort à son prochain. C'est le premier épisode de la Genèse qui met en présence «la mort», cette mort qu'un des deux hommes donnera à l'autre. Le "masochisme moral » s'inscrirait-il donc du côté du " complexe de Caïn », et le "masochisme sexuel », de celui du "complexe de la Vénus à la fourrure »? Rien n'est moins sûr puisque c'est à une intrication des deux que nous assistons le plus souvent.

Gilles Deleuze ${ }^{4}$ nous donne du masochisme un brillant éclairage qui complète celui de Freud. Freud insistait sur le rapport entre la passivité et l'activité dans le couple sado-masochiste en différenciant clairement le sadique d'un côté et le masochiste de l'autre. Mais la clinique nous indique le retournement des fonctions et surtout l'existence d'un authentique contrat qui est passé entre le masochiste et son bourreau qui est aussi sa victime.

On se souvient de ce très beau film de Joseph Losey, The Servant, une brillante démonstration de la dialectique de Hegel sur le rapport entre le maître et l'esclave. C'est un travail appliqué du trotskisme de Losey qui conduit la démonstration du cinéaste mais ce cinéaste possède une âme et quelle âme ! C'est l'analyse des méandres de " l'appareil de l'âme » que va disséquer Joseph Losey au moins autant, si ce n'est plus, que la dialectique marxiste. Les personnages troubles du scénario nous laissent comprendre que, plus que le plaisir de l'emprise narcissique, c'est la pérennité d'un contrat moral que désire le masochiste. Le masochiste n'a de cesse d'entraîner l'autre dans la contemplation et la complicité de son acharnement à se défaire lui-même. C'est là que l'un et l'autre, le masochiste et son complice, se retrouvent dans un but commun, un dénominateur commun : une prise de risque de sa propre identité, cette prise de risque dont l'éprouvé toxique demeure le but ultime. La prise de risque est le moment d'un éprouvé sensoriel qui alimente la pulsion elle-même. C'est bien là que réside l'effet apparemment contradictoire de la décharge pulsionnelle : $c^{\prime}$ est en se déchargeant que la pulsion s'alimente et se recharge. C'est cette circularité dynamique d'une autorégénération de la pulsion qui implique la nécessité de la répétition de sa satisfaction. C'est dans cette " compulsion à la répétition ", dans cet effet accompli par la pulsion de mort, que le sujet trouve l'énergie pour se maintenir en vie. Et dans ces effets de répétition, un dénominateur commun apparaît invariablement et ce dénominateur est la prise de risque.

3. R. Sacher-Masoch, Le legs de Caïn, Paris, In libro veritas, 1975.

4. G. Deleuze (2005), Présentation de Sacher-Masoch, Paris, Éditions de Minuit, 2007. 
Ceci m'avait amené lors d'un précédent travail à avancer le dénominatif de « risqueur " pour mieux appréhender la problématique addictive ${ }^{5}$. Dirk Bogarde passe de la peau d'un Barrett soumis, à celle d'un terrible maitre indispensable pour assurer la survie psychique d'un Tommy joué par James Fox. Pinter a signé un scénario où il apparaît que le maître et son servant sont unis par un authentique contrat moral, un contrat moral alimenté par le sexuel.

La trame est faite d'impasses et de répétitions qui ne sont en fait que les figures des articles d'un contrat passé entre le masochiste et l'objet qui alimentera sa pulsion. Car c'est bien d'un contrat dont il s'agit et nous allons maintenant tenter d'en saisir les termes et la visée profonde en quittant les salles obscures des cinémas pour nous transporter dans des bars dits " réservés », dans certains de ces bars que la communauté gay fréquente assidûment sur tous les continents : les bars où les adeptes du « bareback » se retrouvent : les bars de la barbaque du bareback.

La barbaque, chacun le sait, est un morceau de viande plus ou moins goûteux. Mais d'où vient cette dénomination ? Lorsqu'une bête est rôtie sur une broche afin d'être consommée, on dit que c'est un barbecue. Barbecue n'est pas un dérivé de l'anglo-saxon mais trouve son origine dans la langue française. C'est lorsque l'on a empalé la bête à rôtir, c'est-à-dire qu'on lui a enfoncé une barre par le cul, qu'on parle de barbecue. La barbaque est une viande qui a été empalée... La barbaque peut être très goûteuse...

Et puis, il y a le bareback. Le bareback est, à l'origine, une pratique équestre. Il s'agit pour le cavalier de monter sans selle, de monter à cru. La comtesse Isabelle de Noailles, grande figure de l'intelligentsia du début du siècle dernier, a laissé d'elle l'image d'une femme exceptionnellement intelligente et cultivée mais aussi celle d'une femme excentrique qui chevauchait nue et à cru dans ses terres d'Oise, non loin des écuries de Chantilly. La fantasmatique sexuelle fit alors tourner les rotatives et les têtes. La pornographie zoophilique imaginée faisait alliance avec la plus prestigieuse noblesse française non sans défrayer la chronique.

Depuis que l'homme a compris qu'il pouvait faire du cheval son ami - nous dirions plutôt : son serviteur -, l'homme dut alors prendre le risque de l'enfourcher pour le domestiquer. L'homme dut oser monter à cru pour pouvoir se déplacer plus vite en déployant moins d'efforts. Le cavalier serait resté bien démuni s'il n'avait pas su inventer des manufactures pour bien asseoir sa domination sur une bête qui, lorsqu'elle est adulte, pèse entre 500 et 1000 kilos. Ces manufactures s'appellent des « aides artificielles ». Donc l'homme inventa les selles, harnais, étriers, rennes, œillères et autres harna-

5. E. Toubiana, « La guerre est-elle un jeu », Topique, n 102, 2008, p. 163-180. 
chements pour rendre cette nouvelle amitié plus rassurante, ou, en d'autres termes, cet asservissement mieux assuré. L'homme disposait déjà de ses " aides naturelles », qui sont les mains, les jambes, le poids du corps. L'inventivité de l'espèce humaine pallia la relative efficacité de ce que la nature lui avait octroyé.

L'art équestre, qui se veut noble, prescrit à l'homme de respecter sa monture et de se plier à un code moral visant au respect de la monture asservie. Et lorsque l'homme se doit de prescrire un code, c'est toujours afin de mieux réguler ses propres débordements. Prenons l'exemple d'un des raffinements inventés par l'homme pour mieux maîtriser sa monture : la « martingale ». La martingale est une sorte de lanière de cuir qui est rivée à une sangle, sous le ventre du cheval, et qui rejoint une muserolle puis le mors. Le mors est cette barre de fer placée dans la bouche du cheval. L'utilisation de la martingale est bien réglementée. Elle sert à mener la bête là où le cavalier veut la conduire. La plus redoutable des martingales est la «martingale fixe ", celle qui contraint le cheval à baisser la tête. La martingale à anneaux n'est pas mal non plus mais elle est principalement réservée au saut d'obstacle cette pratique équestre où il est primordial de s'assurer de la complète obéissance de la monture. Eh oui, nous avions oublié que la martingale n'est pas simplement un artifice que les joueurs de casino connaissent fort bien ! Si la martingale est la première aide mise à la disposition du cavalier pour ne pas se laisser démonter par sa monture, elle est pour le joueur l'espoir de faire sauter la banque du casino. Il n'existe aucune martingale imparable pour faire sauter la banque. Aucun cavalier ne peut se vanter de n'avoir jamais chuté. Le cavalier n'est pas mécontent d'exhiber les traces de ses nombreuses fractures qu'il qualifie comme autant de décorations.

Les cow-boys célèbrent le bareback. C'est une activité ludique, un sport, qu'ils pratiquent sur des chevaux sauvages et dont l'enjeu consiste à pouvoir rester sur le dos de la bête le plus longtemps possible et ce, sans aucune aide ni martingale. Des championnats, où de fortes sommes d'argent sont pariées, sont régulièrement organisés et déplacent bon nombre d'amateurs. Tenir le plus longtemps possible sur des montures les plus rétives possible, et la gloire d'un jour est au bout de la cascade de ruades qu'il a fallu subir et à laquelle il a fallu survivre. Des cavaliers risquent leur vie à ce jeu-là ! Ils y gagnent la gloire en plus de leurs émoluments. Les chevaux indomptés entrent dans la légende. Les indomptables gagneront leur place dans des prés paisibles, loin des arènes guerrières.

Mais, ces dernières années, le « bareback » est devenu aussi une pratique sexuelle gay, en expansion constante. Elle s'est même institutionnalisée. Le bareback est un jeu dont la règle est simple : avoir des relations sexuelles non protégées avec des partenaires susceptibles de porter le virus VIH. Le bare- 
back est un véritable jeu de roulette russe mais ici, c'est le pénis en érection qui est la seule arme. Une seule cartouche, une seule relation sexuelle, une seule éjaculation, et la partie est jouée. Le trophée convoité n'est rien d'autre que le sida. C'est le nec plus ultra pour certains homosexuels qui n'ont qu'un profond mépris envers ceux qui se préservent. «Act up » est le plancher des vaches des pilotes d'essais que sont nos risqueurs du bareback.

Les risqueurs, ceux qui témoignent maintenant publiquement de l'intensité de la jouissance que cette pratique génère et du désir de la répéter le plus souvent possible, forment une véritable congrégation, une élite parmi l'élite. Les barebackers sont devenus dépendants de leurs pratiques. Certains l'assimilent à une véritable addiction, une activité dont ils ne pourraient se passer. Et là, pas de martingales !

Dans un premier temps les choses pourraient paraître simples puisqu'il y a d'un côté le bug chaser (le chasseur de parasite) et le gift giver (le donneur $\mathrm{du}$ cadeau). Les choses sont plus complexes lorsque l'on comprend que, comme dans un bal masqué, on ne sait pas qui est le donneur et qui est le chasseur. Les choses atteignent le paroxysme du paradoxe lorsque l'on ne sait pas si le but à atteindre est d'être contaminé, d'éviter de l'être, ou d'endosser la gloire d'avoir transmis le virus.

La jouissance réside simplement, le plus simplement du monde, dans l'éprouvé vertigineux du risque de gagner ou de perdre. Une nique à la mort s'impose, plus que sa quête. Initialement, cette pratique excentrique de la sexualité homosexuelle ne s'assimile, pour les barebackers, ni à une conduite perverse, ni à une mise en scène meurtrière, ni à une tentative suicidaire. Certains auteurs ont voulu en effet apparenter la pratique du bareback à l'actualisation d'une problématique mélancolique ou d'une mise en acte psychopathique et perverse. Les barebackers ne se vivent pas comme des pervers puisqu'ils désignent eux-mêmes le pervers comme celui qui désire transmettre le virus sans que le protagoniste sache qu'il y a effectivement un risque qu'il soit contaminé. Ce n'est pas le cas dans ce cadre strict du jeu du bareback. Le vrai barebacker est, se veut, et reste dans la logique d'un contrat cosigné. Ce n'est pas un auto-anéantissement qui est visé par la poussée pulsionnelle, mais au contraire une assurance d'existence éprouvée pleinement grâce à cette prise de risque partagée par deux sujets.

$C^{\prime}$ est à partir de ce comportement toxique si particulier que nous allons maintenant tenter de considérer les points communs que nous rencontrons dans la clinique des patients qui souffrent d'autres addictions. Les barebackers dont nous venons de parler ne souffrent pas réellement de leurs passions. Lorsqu'ils sont amenés à consulter, ce n'est pas nous qu'ils viennent voir mais plutôt le généraliste ou un centre spécialisé dans la détection du virus du sida. 
Par contre, la singularité avec laquelle ils vivent leurs dépendances au risque d'être contaminés présente quelques analogies avec la mise en œuvre des autres problématiques addictives, qu'elles soient avec ou sans toxiques. Nous assistons le plus souvent à une réification de l' « objet toxique » et l'addicté entretient avec lui le même type de relation que le bug chaser et son gift giver.

Je poserais l'hypothèse que les personnalités dites « addictives » nous donnent à voir un spectacle analogue à celui de notre cow-boy dont la raison de vivre dépend du risque compulsif qu'il prend à chevaucher une monture dont il sait qu'il ne peut attendre le meilleur qu'en côtoyant le pire. Notre cow-boy ne cherche pas la mort. Notre cow-boy ne veut que se mesurer avec le risque qu'elle advienne éventuellement. C'est la prise de risque qui reste pour lui le moteur de sa pulsion.

Pour l'addicte, le souci n'est-il pas de nous donner à voir la mise en danger dans laquelle ils se mettent ou ils mettent l'autre ? Ce donner à voir n'est pas sans rappeler l'exhibitionnisme du pervers meurtrier qui trouverait autant de jouissance à accomplir son acte qu'à le voir démontrer et démonter devant son public ${ }^{6}$. Est-ce un effet de leur propre volonté d'autonéantisation qui est en œuvre, ou plutôt l'incapacité dans laquelle ils se trouvent de se passer de leurs toxiques sinon à courir le risque d'un effondrement psychique ou somatique ? Ce patient addicté nous donne-t-il à voir son addiction, ou n'est-ce pas plutôt une cicatrice morale qu'il exhibe en rendant le destin ou le hasard coupable d'une série de circonstances qui l'a conduit à ce tel épuisement psychique et somatique?

$C^{\prime}$ est, nous en posons l'hypothèse, à cette monstration que nous assistons lorsque nous nous trouvons pris dans les rets de la prise en charge d'un patient qui souffre d'addiction. D'emblée, le masochiste a subtilisé la signature du clinicien en lui faisant insidieusement signer le contrat. La signature du contrat réside dans cet énoncé : "Quel masochisme ! » Sortis de notre bureau d'entretien ou de la chambre de certains patients assez mal en point pour devoir rester en chambre, nous rendons rapidement compte de notre premier sentiment clinique en le ponctuant le plus souvent par ce «quel masochisme!».

Que ces patients soient des alcooliques, des cocaïnomanes, des héroïnomanes, des accrochés de joints de haschisch, ou des joueurs invétérés et déprimés par l'abîme de leurs pertes, le praticien observe toujours chez eux une mise en scène savamment articulée, même à leur insu. Notre patient exhibe sa légion d'honneur de sa défaite. Dans l'armée on appelle ça la dégradation. Fractures, yeux au beurre noir, pansements protégeant les

6. M.L. Susini, L'auteur du crime pervers, Paris, Fayard, 2002. 
plaies et contusions multiples, sont les décorations piteusement attribuées à des coins de portes, à des placards aux tiroirs ouverts, à des chutes d'escabeau mal assuré, à des accidents de voiture subis ou provoqués. Ce peuvent être aussi des entailles aux poignets, des traces de quelques suicides ratés comme tout le reste d'ailleurs.

L'énoncé faussement diagnostique de " masochisme » est tombé comme une sentence qui n'est rien d'autre que la sanction désirée. Le mépris ou la compassion de l'autre sont autant de signes d'une reconnaissance qu'un réel combat a bien lieu entre le patient et son toxique. Le cheval peut être identifié comme étant un pharmakon comme les autres pharmakons. Il peut être au service du plaisir de l'homme mais il peut tout aussi bien se révéler être un redoutable toxikon au service de sa chute. Le praticien est en position de spectateur, de celui qui voit ce que l'autre exhibe. Ce que l'autre exhibe, c'est la démonstration de sa farouche volonté de vivre malgré les piètres artifices qui lui assurent sa survie.

Le sujet addicté n'a d'autre choix que de se complaire dans son dangereux exercice de funambule, de cascadeur ou de barebacker. C'est la seule « formation de compromis » qu'il a trouvée comme assurance de survie. Il est devenu banal de lire que la relation transférentielle avec le patient addicté n'est qu'un leurre. Nous ne pouvons que souscrire à cette banale proposition puisque ce qui importe avant tout pour notre patient, c'est la relation mortifère dont il jouit grâce à son toxique et l'insistance avec laquelle il la montre. Là réside l'imposture première.

Lorsqu'il arrive que le thérapeute quitte cette position de simple témoin (un témoin plus ou moins efficace...) pour devenir à son tour l'objet privilégié de la relation qu'entretient l'addicté avec le monde, ce thérapeute peut être certain qu'il aura à occuper une fonction bien particulière : monter à cru sur un cheval bien rétif ou être lui-même une monture que l'autre n'aura de cesse de convaincre du caractère nécessaire du tumulte de la rencontre, des chutes, et des rechutes.

Rechutes, répétition de même, travail de la pulsion dans ce qui la caractérise, son exigence interne de répétition mortifère, telles sont les immuables mises en scène «données à voir » et même « données à vivre », tant la posture analytique s'avère comparable à ces scènes de rodéo où les situations de la monture se confondent avec celles de son cavalier dans cette fascination de cet obscène d'être pour la mort. 


\section{BIBLIOGRAPHIE}

Deleuze, G. 2006. Présentation de Sacher-Masoch, Paris, Les Éditions de Minuit, 2007. FREUD, S. 1927. L'avenir d'une illusion, Paris, PUF, 1971.

KRAFFT-EBING, 1886. Psychopathia sexualis, Paris, Payot, 1960.

SACHER MASOCH, R. 1975. Le legs de Caïn, Paris, In libro veritas.

SACHER-MASOCH, R. 1976. La Vénus à la fourrure, Paris, Les Mille et une nuit.

SusINI, M-L. 2002. L'auteur du crime pervers, Paris, Fayard.

ToubianA, E. 2008. « La guerre est-elle un jeu », Topique, n 102, p. 163-180.

Résumé

La clinique des addictions révèle les écueils et impasses dans lesquels le praticien se retrouve en position de spectateur d'un scénario d'où il se trouve exclu en tant que sujet. Ses élaborations et ses tentatives de se maintenir dans une posture qui serait autre que celle d'un simple voyeurisme l'engage, s'il n'y prend garde, à se confondre dans un exercice équestre bien loin de celui préconisé par Ferenczi, un exercice où non pas la mort mais l'exigence de sa proximité démontre le travail mis en œuvre par la pulsion.

Mots-clés

Addictions, barbaque, Bareback, risque, répétitions, exhibition, imposture.

BAREBACK FLESH :

UNCOMFORTABLE POSTURES.

\section{Summary}

The clinical study of addiction reveals obstacles and impasses in which the clinician finds him or herself the spectator to a scenario from which he or she is excluded as a subject. These manifestations and attempts to maintain a posture other than that of simple voyeurism engages, if one is not careful, with an equestrian exercise far from that recommended by Ferensky, an exercise where not death itself, but the demands of its proximity demonstrate the work set in motion by the drive.

Keywords

Addictions, flesh, Bareback, drive, repetition compulsion, exhibition, imposture. 